\title{
Low-Luminosity Accretion in Black Hole X-Ray Binaries and Active Galactic Nuclei
}

\section{Citation}

Narayan, Ramesh. 2005. "Low-Luminosity Accretion in Black Hole X-Ray Binaries and Active Galactic Nuclei." Astrophysics and Space Science 300 (1-3): 177-88. https://doi.org/10.1007/ s10509-005-1178-7.

\section{Permanent link}

http://nrs.harvard.edu/urn-3:HUL.InstRepos:41384920

\section{Terms of Use}

This article was downloaded from Harvard University's DASH repository, and is made available under the terms and conditions applicable to Other Posted Material, as set forth at http:// nrs.harvard.edu/urn-3:HUL.InstRepos:dash.current.terms-of-use\#LAA

\section{Share Your Story}

The Harvard community has made this article openly available.

Please share how this access benefits you. Submit a story.

Accessibility 


\title{
Low-Luminosity Accretion in Black Hole X-ray Binaries and Active Galactic Nuclei
}

\author{
Ramesh Narayan (narayan@cfa.harvard.edu) \\ Harvard-Smithsonian Center for Astrophysics, 60 Garden Street, Cambridge, MA \\ 02138, U.S.A.
}

\begin{abstract}
At luminosities below a few percent of Eddington, accreting black holes switch to a hard spectral state which is very different from the soft blackbody-like spectral state that is found at higher luminosities. The hard state is well-described by a two-temperature, optically thin, geometrically thick, advection-dominated accretion flow (ADAF) in which the ions are extremely hot (up to $10^{12} \mathrm{~K}$ near the black hole), the electrons are also hot $\left(\sim 10^{9-10.5} \mathrm{~K}\right)$, and thermal Comptonization dominates the X-ray emission. The radiative efficiency of an ADAF decreases rapidly with decreasing mass accretion rate, becoming extremely low when a source reaches quiescence. ADAFs are expected to have strong outflows, which may explain why relativistic jets are often inferred from the radio emission of these sources. It has been suggested that most of the X-ray emission also comes from a jet, but this is less well established.
\end{abstract}

Keywords: accretion, accretion disks - active galactic nuclei — black hole physics — radiation mechanisms - X-rays: binaries

\section{Introduction}

The well-known thin accretion disk model has been a staple of accretion theory for more than 30 years (Shakura \& Sunyaev 1973; Novikov \& Thorne 1973). It provides a good description of the soft X-ray spectra of luminous black hole X-ray binaries (XRBs) in the high soft state (see McClintock \& Remillard 2004) and the big blue bump in the optical/UV spectra of bright quasars and active galactic nuclei (AGN; Malkan 1983; but see Koratkar \& Blaes 1999). However, even from the earliest days (Tananbaum et al. 1972) it was realized that XRBs sometimes switch to a hard spectral state which requires the accreting gas to be hot and optically thin, quite different from the gas in a thin disk which is relatively cool and optically thick.

Observations of a number of XRBs have shown that, at luminosities below a few percent of Eddington, the sources enter the classic low hard state, and at much lower luminosities the quiescent state (McClintock \& Remillard 2004). Both states are characterized by very high temperatures $\sim 100 \mathrm{keV}$ or more, optically thin emission, and weak or absent soft X-ray emission. In the case of supermassive black holes, low-luminosity AGN (LLAGN) are noted for the absence of a

(c) 2018 Kluwer Academic Publishers. Printed in the Netherlands. 
big blue bump and the presence of substantial hard X-ray and radio emission (Ho 1999; Quataert et al. 1999; Nagar et al. 2000). This again indicates that a standard thin accretion disk is either absent or is energetically unimportant, and that a hot flow, similar to those seen in low-luminosity XRBs, is probably present.

In an important paper, Shapiro, Lightman \& Eardley (1976) introduced the idea of a two-temperature plasma and used it to develop a new hot accretion solution which is distinct from the standard thin disk. However, the solution turned out to be thermally unstable (Pringle 1976). Fortunately, there is a second hot two-temperature solution called an advection-dominated accretion flow (ADAF; Narayan \& Yi 1994, 1995b; Abramowicz et al. 1995; see Narayan, Mahadevan \& Quataert 1978; Kato, Fukue \& Mineshige 1998 for reviews). This solution, which is also referred to as a radiatively inefficient accretion flow (RIAF), was originally discussed in a forgotten paper by Ichimaru (1977; see also Rees et al. 1982). It has been shown to be effectively stable (Kato et al. 1997; Wu 1997), and it is now recognized to be relevant for understanding low-luminosity accretion flows around black holes.

\section{Advection-Dominated Accretion Flow}

\subsection{BASIC Properties}

The energy equation of gas in a time-steady accretion disk may be written schematically as

$$
q_{\mathrm{adv}} \equiv \rho v \frac{T d s}{d R}=q_{+}-q_{-},
$$

where $q_{\mathrm{adv}}$ is the rate of advection of energy per unit volume, $\rho$ is the density, $v$ is the radial velocity, $T$ is the temperature, $s$ is the specific entropy, $R$ is the radius, $q_{+}$is the viscous heating rate per unit volume, and $q_{-}$is the radiative cooling rate. A thin accretion disk is characterized by the condition $q_{+} \sim q_{-} \gg q_{\text {adv }}$, i.e., viscous heating is balanced by radiative cooling. In contrast, an ADAF satisfies $q_{+} \sim$ $q_{\text {adv }} \gg q_{-}$, i.e., most of the viscous heat remains trapped in the gas (because the gas is radiatively inefficient), and the energy is advected in towards the $\mathrm{BH}$. Technically, since the plasma is two-temperature, it is necessary to write separate energy equations for the ions and the electrons and to model the energy transfer between the two species by Coulomb collisions (Narayan \& Yi 1995b; Nakamura et al. 1997). We do not go into the details here and refer the reader to the review by Narayan et al. (1998). 
The ADAF solution has a number of interesting properties:

(i) The ion temperature varies roughly as $T_{i} \sim 10^{12} \mathrm{~K} / r$, where $r=$ $R / R_{S}$ is the radius in Schwarzschild units. The electron temperature, however, saturates at $T_{e} \sim 10^{9}-10^{10.5} \mathrm{~K}$ for $r \lesssim 10^{2}-10^{3}$.

(ii) The large ion temperature implies that the flow is geometrically thick. In fact, an ADAF might be viewed as the viscous rotating analog of spherical Bondi accretion.

(iii) The gas in an ADAF is optically thin; therefore, the radiation from the hot electrons (which dominate the emission) is primarily by thermal Comptonization. Because Comptonization acts as a natural thermostat, the electron temperature is typically $\sim 100$ to a few 100 $\mathrm{keV}$ and varies by only a factor of a few over a wide range of Eddingtonscaled accretion rate $\dot{m}=\dot{M} / \dot{M}_{\text {Edd }}$ (e.g., Esin, McClintock \& Narayan 1997; Esin et al. 1998; Zdziarski et al. 2003).

(iv) The ADAF solution exists only for accretion rates $\dot{m}$ below a certain critical rate $\dot{m}_{\text {crit }}$, whose value depends on the viscosity parameter $\alpha$. For $\alpha \sim 0.1-0.25, \dot{m}_{\text {crit }} \sim 0.01-0.1$.

\subsection{Applichtion of the ADAF Solution to XRBs and AGN}

Rather miraculously, the properties of the ADAF solution are exactly what are needed to understand XRBs at low luminosities. The radiation in an $\mathrm{ADAF}$ is dominated by thermal Comptonization, in agreement with observations of XRBs in the low hard state. The electron temperature is about $100 \mathrm{keV}$, exactly what is needed to explain X-ray spectra in the low state (e.g., see the ADAF model of GRO J0422+32 shown in Fig. 1). Finally, the critical $\dot{m}_{\text {crit }} \sim 0.01-0.1$ above which the ADAF solution ceases to exist is consistent with the luminosity at which the transition from the low hard state to the high soft state occurs in XRBs.

By combining the thin accretion disk model and the ADAF model, Narayan (1996) and Esin et al. (1997) showed that it is possible to understand qualitatively the various spectral states of XRBs. According to their proposal (Fig. 2), for $\dot{m}>\dot{m}_{\text {crit }}$, the accretion occurs primarily via a thin disk with a corona on top. This corresponds to the high soft state, with the disk providing the bulk of the radiation via a multicolor blackbody component and the corona contributing hard X-rays through Compton scattering (Haardt \& Maraschi 1991). Once $\dot{m}$ falls below $\dot{m}_{\text {crit }}$, a hole opens up at the center of the disk and the hole is filled with a hot ADAF. For $\dot{m} \lesssim \dot{m}_{\text {crit }}$, the hole is relatively small and both the thin disk and the ADAF contribute roughly equally. This corresponds to the intermediate state. With decreasing $\dot{m}$, the transition radius $r_{\mathrm{tr}}$ between the two zones becomes larger and the ADAF dominates the 


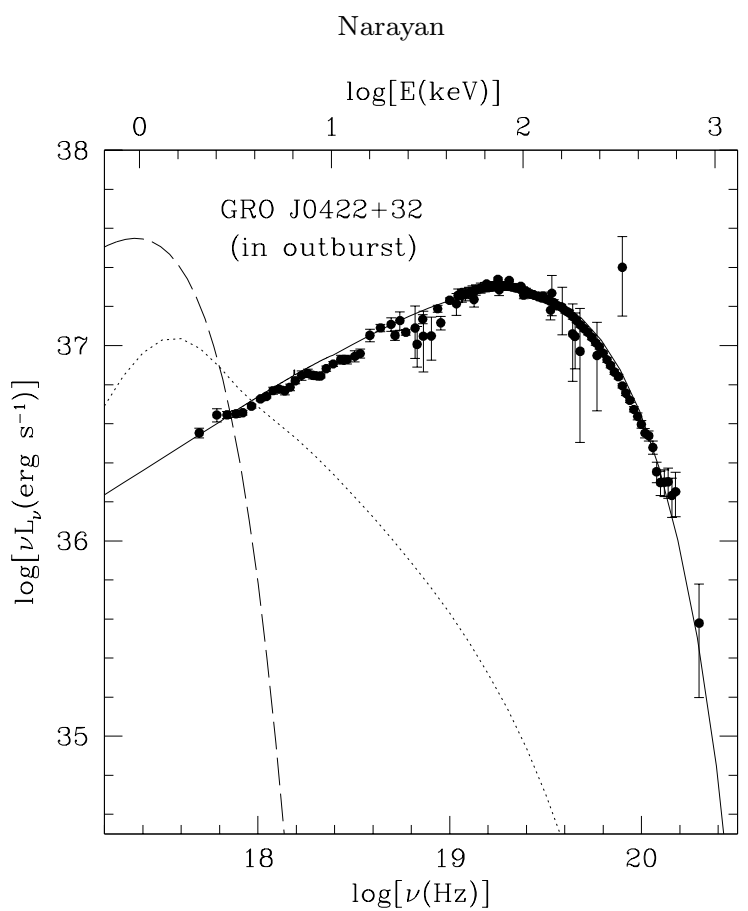

Figure 1. Combined TTM (2-20 keV), HEXE (20-200 keV), and OSSE (50-600 $\mathrm{keV}$ ) spectrum of GRO J0422+32 in the low hard state. The solid line shows an ADAF fit to the spectrum. (From Esin et al. 1998)

energetics. At very low $\dot{m}$, i.e., in the quiescent state, $r_{\text {tr }}$ is very large (> 1000, e.g., Narayan, McClintock \& Yi 1996); in objects such as Sgr A* (Yuan, Quataert \& Narayan 2003), the outer thin disk may even disappear altogether.

In the intermediate state and at the high end of the low state, the ADAF is only mildly advection-dominated, so the radiative efficiency is fairly large. However, with decreasing $\dot{m}$, the efficiency drops rapidly. Quiescent systems are, therefore, radiatively very inefficient; Sgr A*, for instance, has a luminosity that is only about $10^{-5}-10^{-6}$ of the rate at which rest mass energy accretes from its surroundings (Yuan et al. 2003).

An ADAF has two sources of soft photons for Comptonization, and both are included in models (Narayan, Barret \& McClintock 1997): (i) thermal synchrotron photons from the hot electrons in the ADAF, (ii) thermal blackbody photons from the outer thin disk. The former dominates at low $\dot{m}$ (quiescent state and lower end of low state) and the latter at higher $\dot{m}$ (upper end of low state and intermediate state).

The above paradigm, which is based on the ADAF model or its variants (ADIOS, Blandford \& Begelman 1999; LHAF, Yuan 2001, Yuan \& Zdziarski 2004; CDAF, Narayan, Igumenshchev \& Abramowicz 


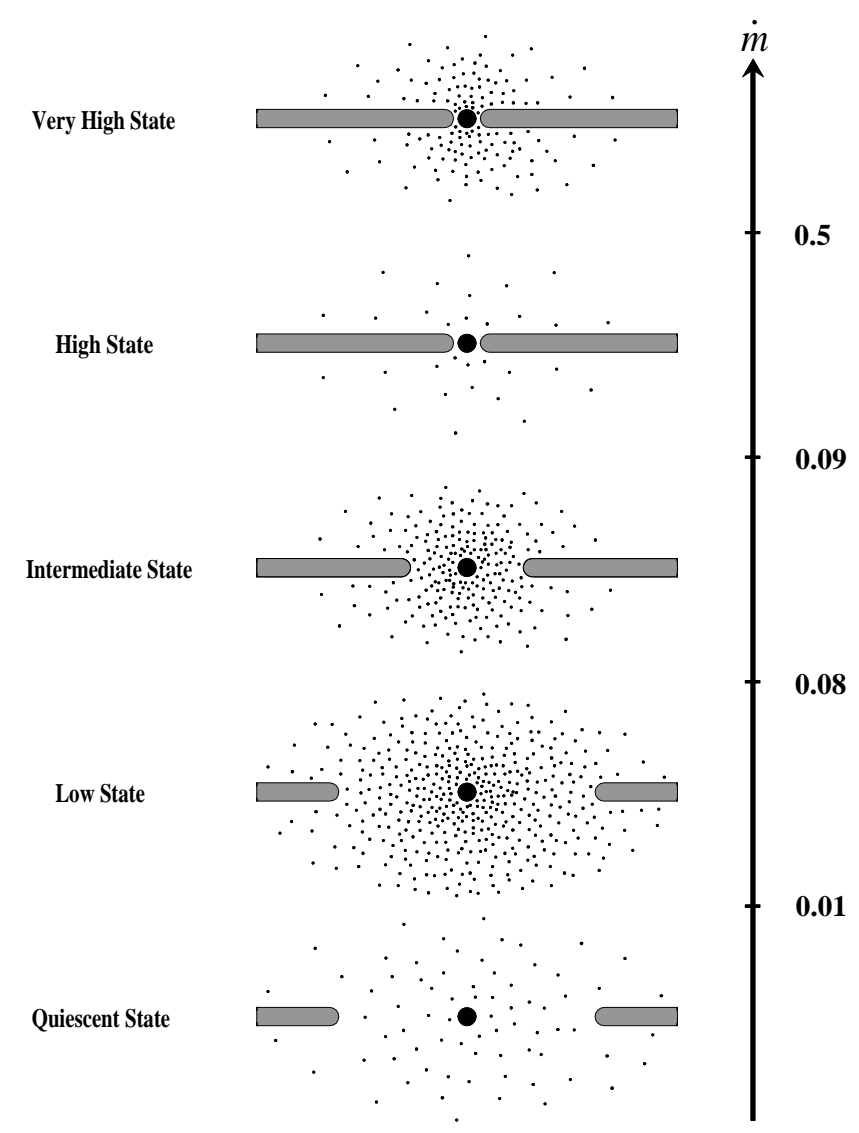

Figure 2. Configuration of the accretion flow around a black hole in different spectral states, shown schematically as a function of the Eddington-scaled mass accretion rate $\dot{m}$. The ADAF and the corona are indicated by dots and the thin disk by the shaded horizontal bars. (From Esin et al. 1997)

2000, Quataert \& Gruzinov 2000), explains qualitatively many observations of XRBs (Esin et al. 1997, 1998, 2001; see Narayan et al. 1998 for other applications). Very recently, Meyer-Hofmeister, Liu \& Meyer (2004) have suggested an interesting mechanism involving an interplay between Compton cooling and disk evaporation to explain the hysteresis phenomenon that has been identified in the high-to-low transition of black hole and neutron star X-ray binaries (Miyamoto et al. 1995; Nowak, Wilms \& Dove 2002; Maccarone \& Coppi 2003; Zdziarski et al. 2004).

The ADAF model also explains a variety of observations of LLAGN: Sgr A* (Narayan, Yi \& Mahadevan 1995; Yuan et al. 2003), LLAGN in giant ellipticals (Fabian \& Rees 1995; Reynolds et al. 1996; Di Matteo 
et al. 2003), and LINERs (Lasota et al. 1996; Quataert et al. 1999). In addition, it appears that ADAFs may be present in BL Lac objects (Maraschi \& Tavecchio 2003), FR I sources (Reynolds et al. 1996; Begelman \& Celotti 2004), XBONGs (Yuan \& Narayan 2004), and even some Seyferts (Chiang \& Blaes 2003). Overall, the model has turned out to be quite useful for providing a qualitative understanding of a variety of phenomena in low-luminosity accreting black holes (Quataert 2001; Narayan 2002).

\subsection{Transition Radius}

A key element of the model shown in Fig. 2 is that the transition radius $r_{\text {tr }}$ between the outer thin disk and the inner ADAF varies with $\dot{m}$. But how exactly does it vary? To calculate this from first principles, one needs a physical theory of what causes the transition between the two kinds of flow. A number of ideas have been discussed in the literature and many efforts have been devoted to estimating $r_{\mathrm{tr}}(\dot{m})$ theoretically (Meyer \& Meyer-Hofmeister 1994; Dullemond \& Turolla 1998; Liu et al. 1999; Rozanska \& Czerny 2000; Spruit \& Deufel 2002), but no model is presently able to provide robust predictions.

An alternative approach is to use the observations themselves to determine $r_{\operatorname{tr}}(\dot{m})$. For a number of sources, by fitting observations one is able to obtain estimates of the Eddington-scaled luminosity and mass accretion rate, as well as the transition radius. Although there are large uncertainties in some of these quantities, nevertheless the results are interesting when plotted, as discussed in Narayan et al. (1998) and Yuan \& Narayan (2004). Figure 3 from the latter paper supports the basic features of the Esin et al. (1997) proposal; specifically, the transition radius $r_{\text {tr }}$ seems to increase monotonically with decreasing luminosity, as postulated in the model. Interestingly, both XRBs and LLAGN are included in the plot, and the two classes of sources seem to follow more or less the same trend even though their masses are very different. This confirms that the physics of ADAFs is largely mass-independent (Narayan \& Yi 1995b), once all quantities are scaled suitably in terms of Eddington and Schwarzschild units.

\section{Role of Jets}

\subsection{ADAFs, Convection, Outflows, Jets}

One of the interesting properties of ADAFs, highlighted already in the first papers (Narayan \& Yi 1994, 1995a), is that the accreting gas has a positive Bernoulli parameter, i.e., the gas is technically not bound 


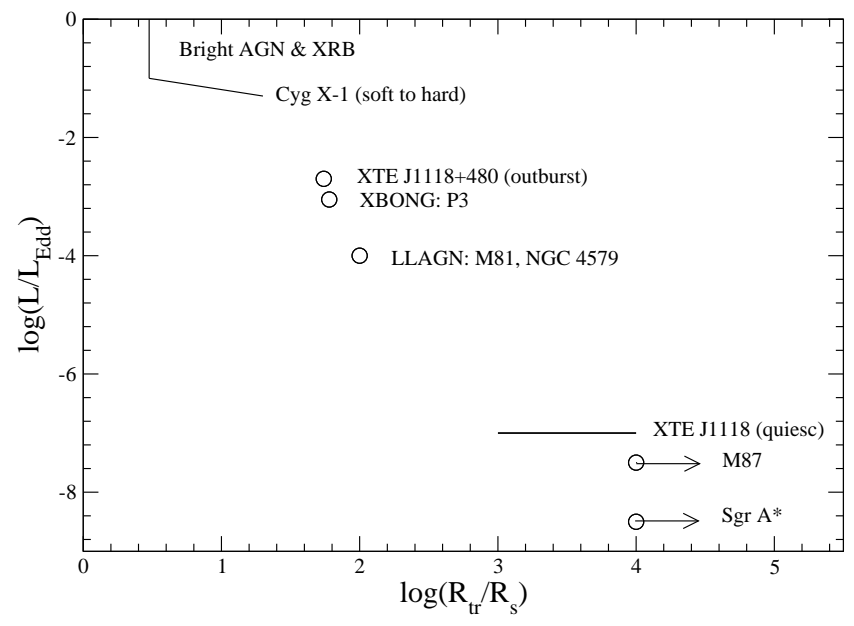

Figure 3. Bolometric luminosity in Eddington units (ordinate) vs. the transition radius in Schwarzschild units (abscissa) for different observed systems. (See Yuan \& Narayan 2004 for details)

to the BH. One expects, therefore, strong winds and outflows from an ADAF (Narayan \& Yi 1994, 1995a). Another property of ADAFs is that they have unstable entropy gradients and are hence violently unstable to convection (Begelman \& Meier 1982; Narayan \& Yi 1994, 1995a). Both effects have been seen in hydrodynamic and MHD simulations of ADAFs (Stone, Pringle \& Begelman 1999; Igumenshchev \& Abramowicz 2000; Narayan et al. 2000; Stone \& Pringle 2001; Hawley \& Balbus 2002; Igumenshchev, Narayan \& Abramowicz 2003).

An important consequence of the above effects is that the mass that accretes onto the $\mathrm{BH}$ via an ADAF is much less than the mass supplied at the outer edge of the accretion flow. Blandford \& Begelman (1999) suggested that the mass accretion rate may scale with radius as $\dot{m} \sim\left(r / r_{\text {out }}\right)^{s}$. This scaling has been widely used in ADAF models (e.g., Quataert \& Narayan 1999). One problem is that the value of $s$ cannot be estimated from first principles, though one may be able, in favorable cases, to fit $s$ by comparison to observations. Yuan et al. (2003) estimated $s \sim 0.3$ for the accretion flow in Sgr A*. For this choice of $s$, the unusually low luminosity of the source is explained partly by the reduced mass accreting on the black hole $\left(\sim 10^{-2}\right.$ of the mass available at the Bondi radius) and partly by the low radiative efficiency of the accreting gas $\left(\sim 10^{-3}\right)$.

Once we recognize that ADAFs have powerful outflows, it is natural to think that these flows would have relativistic jets (Meier 2001). Indeed, such a connection has been established fairly convincingly. XRBs in the low state generally have measurable radio emission, whereas 
sources in the high state do not. The radio emission has been resolved into a jet in Cyg $\mathrm{X}-1$, and jets are inferred in other sources because of their large brightness temperatures (Fender 2004). In the case of supermassive BHs again, it is found that LLAGN are in general radio loud with high brightness temperatures (Nagar et al. 2000; Falcke et al. 2000). Also, BL Lacs, which have been associated with ADAFs (Maraschi \& Tavecchio 2003), are known to have strong jets. Apart from these experimental indications, there is also a strong theoretical argument for jets, viz., an ADAF simply cannot produce the large radio fluxes that are observed. The radio emission has to come from a volume much larger than the ADAF, which suggests that it must originate in a jet.

\subsection{Does the Jet Dominate the High Energy Emission in ADAFs?}

While the argument for the radio emission originating in a jet is clear, what about the X-ray emission? The ADAF model is quite successful in explaining the X-ray fluxes and spectra of low-luminosity black holes without invoking a jet (e.g., Fig. 1). One source of particular interest is XTE J1118+480, for which a nearly complete spectrum has been measured in the low state (McClintock et al. 2001). Esin et al. (2001) proposed a model for this source in which (as in Fig. 2) a thin disk is present outside a transition radius $r_{\mathrm{tr}} \sim 50$ and an ADAF is present inside this radius. The model fits the spectral data in the optical, UV and X-rays quite well.

Soon after this work, Markoff et al. (2001) proposed an alternative model in which they explained the entire spectrum of XTE J1118+480 from radio to X-rays by means of synchrotron emission from a jet. (They invoked a standard disk for the optical and UV.) As described earlier, a jet is certainly expected in an ADAF system and it is quite natural for the jet to dominate in radio and perhaps infrared. What was surprising was that the Markoff et al. model was able to explain the X-ray emission with the same jet.

The case for a jet became stronger when Corbel et al. (2003) showed that there is a strong correlation between the radio and X-ray emission in the black hole XRB GX 339-4 in the low state and quiescent state. They suggested that a significant fraction of the X-ray emission may originate in a jet. Interestingly, both Markoff et al. and Corbel et al. require a radiatively inefficient $\mathrm{ADAF}$ to be present since a radiatively efficient disk would swamp the jet emission in their model. However, the ADAF is postulated to be virtually silent even in the X-ray band, and it is the jet that produces most of the observed radiation. 
Heinz \& Sunyaev (2003) studied the jet model and worked out a scaling relation between the synchrotron flux at a given frequency, the mass of the black hole, and the mass accretion rate. Their model is applicable to jets anchored in either an ADAF or a standard disk. Merloni, Heinz \& di Matteo (2003) extended this work and showed that accreting black holes follow quite well a "fundamental plane" in the three-dimensional parameter space of radio luminosity, X-ray luminosity and black hole mass. However, they came down in favor of the ADAF rather than the jet as the source of the X-ray emission in the low hard state. Falcke, Körding \& Markoff (2004) argued instead that synchrotron emission from the jet is the source of the X-rays. In a recent paper, Heinz (2004) has presented additional arguments why a synchrotron jet is unlikely to explain the X-ray emission in low hard state binaries.

Apart from the above contradictory arguments, Zdziarski et al. (2003) have presented possible additional difficulties with a jet interpretation of the X-ray emission in the low state. They claim that synchrotron emission cannot produce as sharp a cut off at high energies as observed (e.g., see the spectrum shown in Fig. 1). Also, the predicted spectrum is not as hard as the spectra observed in some low state XRBs. Finally, the fact that the cutoff occurs near 100 or a few $100 \mathrm{keV}$ in several sources (in fact, all sources in which a cutoff has been seen) does not find a natural explanation in the jet model; it requires a degree of finetuning of the power-law energy distribution of the radiating electrons. In the ADAF model, on the other hand, thermal Comptonization acts as a thermostat that naturally produces a temperature on the order of $100 \mathrm{keV}$.

Recently, Yuan et al. (2004) and Malzac et al. (2004) have come up with a jet-ADAF model of J1118+480 in which a jet produces most of the radio and infrared emission, the ADAF produces the X-ray emission, and the outer thin disk produces the optical and UV emission. The model fits the spectral data satisfactorily and also explains the timing and variability data qualitatively. By combining the best features of the ADAF model and the jet model, this work appears to represent an interesting compromise between the two models.

\subsection{Is the X-RAy Emission Beamed?}

The most obvious feature of a jet is that it involves outward motion of gas at relativistic speeds. It is therefore natural to expect evidence in the data for relativistic beaming. Observations, however, generally indicate that beaming is not very pronounced. Gallo, Fender \& Pooley (2003) and Fender et al. (2004) estimated an upper limit of $\gamma \sim 2$ 

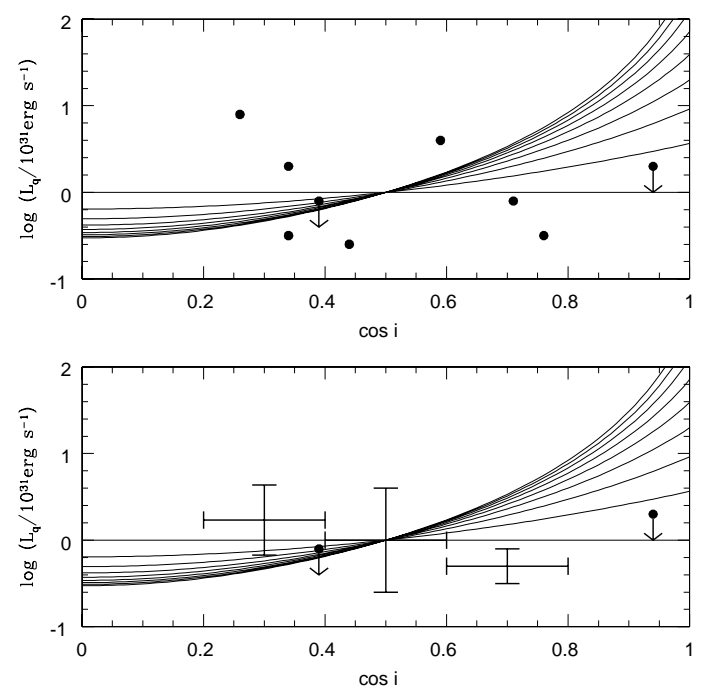

Figure 4. Upper panel: X-ray luminosities of quiescent black hole XRBs in units of $10^{31} \mathrm{erg} \mathrm{s}^{-1}$ plotted against the cosine of the inclination angle $i$. The curves indicate the expected variation according to a jet model for different choices of the jet Lorentz factor: $\gamma=1.0$ (horizontal line), 1.2, 1.4, .., 2.6. Lower panel: The same data grouped into bins of width 0.2 in $\cos i$. (From Narayan \& McClintock 2005)

for the Lorentz factor of the radio-emitting material, while Maccarone (2003) deduced $\gamma \leq 1.4$ for the X-ray-emitting gas in GRO J1655-40.

Figure 4 shows analogous results for the quiescent state of XRBs. Assuming the X-ray emission is from a jet, the different curves show the expected variation of the observed flux as a function of the binary inclination $i$ for different choices of the jet Lorentz factor $\gamma$. The calculations assume that the jet is oriented perpendicular to the binary orbit, and the curves have been normalized so as to have the same flux density for $\cos i=0.5$. Overplotted on the curves are the quiescent X-ray luminosities of a number of black hole XRBs. We see that there is no hint in the data for any increase in the observed luminosity of low-inclination systems $(\cos i \rightarrow 1)$. In fact, the most pole-on system in the sample, $4 \mathrm{U} 1543-47$, with $i \approx 21^{\circ}$, has a $95 \%$ confidence upper limit on its quiescent luminosity that is below the predicted luminosity for all reasonable values of $\gamma$. By visual inspection we conclude that, if the X-ray emission is from a jet, then the Lorentz factor is limited to $\gamma \lesssim 1.2$. A likely explanation for the data is that the X-ray emission is not primarily from an outflowing jet but from an orbiting ADAF. 
Note, however, that the argument assumes the jet to be oriented perpendicular to the accretion disk which is not supported by the limited data available (see Narayan \& McClintock 2005).

Jet models generally have radio emission coming from farther out in the jet and X-ray emission from closer to the center. In fact, often the X-rays are postulated to be emitted from the "base of the jet." In this context, we should note that the base of the jet is probably right inside the ADAF. It then becomes a matter of semantics whether this gas should be called the jet or the ADAF. If the gas were moving rapidly away from the accreting gas and we could see clear evidence for beaming, then we could of course confidently claim that it is a jet. However, as mentioned above, there is no evidence yet for relativistic beaming in either the low state or the quiescent state.

The other distinguishing feature of the jet model is that it invokes synchrotron radiation from nonthermal electrons to explain the Xray emission. The ADAF model, in contrast, makes use of thermal Comptonization. As mentioned above, the synchrotron model has some difficulty explaining certain aspects of the X-ray spectrum. Nevertheless, the arguments are probably not insurmountable, so the model must be considered viable. If, however, it turns out that the synchrotron idea cannot be made to work for the X-ray emission, and if one needs to invoke something like thermal Comptonization in the jet to explain the data, then the argument for the jet would be significantly weakened. Why refer to it as a jet if the gas is located inside the ADAF, is not moving rapidly, and has all the characteristics of the hot gas in an ADAF? For all practical purposes, such a model would be identical to the jet-ADAF model of Yuan et al. (2004) and Malzac et al. (2004) in which the low-energy radio (and infrared) emission comes from a bona fide jet, but the high energy X-ray emission comes from an ADAF. (Malzac et al. also discuss the possibility that the X-rays may come from a patchy corona rather than a standard ADAF.)

One issue still remains to be addressed, viz., the Corbel et al. (2003) correlation between the radio and X-ray emission. This correlation finds a natural explanation in the jet model, but is not so obvious if the $\mathrm{X}$ rays originate in an ADAF. If the jet is part of the general outflow from the ADAF as we have suggested above, then it is conceivable that there would be a correlation between the properties of the jet and those of the ADAF. In this case, even though the radio and the X-rays come from different parts of the system (jet and ADAF, respectively), there might still be a strong correlation between the two. This possibility is discussed in Meier (2001) and needs to be investigated quantitatively. 
The author thanks Tom Maccarone for comments. This work was supported in part by grants NAG5-10780 from NASA and AST 0307433 from NSF.

\section{References}

Abramowicz, M. A., Chen, X., Kato, S., Lasota, J.-P., \& Regev, O. 1995, ApJ, 438, L37

Begelman, M. C., \& Celotti, A. 2004, MNRAS, 352, L45

Begelman, M. C., \& Meier, D. L. 1982, ApJ, 253, 873

Blandford, R. D., \& Begelman, M. C. 1999, MNRAS, 303, L1

Chiang, J., \& Blaes, O. 2003, ApJ, 586, 97

Corbel, S., Nowak, M. A., Fender, R. P., Tzioumis, A. K., \& Markoff, S. 2003, A\&A, 400, 1007

Di Matteo, T., Allen, S. W., Fabian, A. C., Wilson, A. S., \& Young, A. J. 2003, ApJ, 582, 133

Dullemond, C. P., \& Turolla, R. 1998, ApJ, 503, 361

Esin, A. A., McClintock, J. E., \& Narayan, R. 1997, ApJ, 489, 865

Esin, A. A., Narayan, R., Cui, W., Grove, J. E., \& Zhang, S.-N. 1998, ApJ, 505, 854

Esin, A. A., McClintock, J. E., Drake, J. J., Garcia, M. R., Haswell, C. A., Hynes, R. I., \& Muno, M. P. 2001, ApJ, 555, 483

Fabian, A. C. \& Rees, M. J. 1995, MNRAS, 277, L5

Falcke, H., Körding, E., \& Markoff, S. 2004, A\&A, 414, 895

Falcke, H., Nagar, N. M., Wilson, A. S., \& Ulvestad, J. S. 2000, ApJ, 542, 197

Fender, R. P. 2004, in Compact Stellar X-ray Sources, eds. W. H. G. Lewin, M. van der Klis (Cambridge Univ. Press), in press (astro-ph/0303339)

Fender, R. P., Belloni, T. M., \& Gallo, E. 2004, MNRAS, in press (astro-ph/0409360)

Gallo, E., Fender, R. P., \& Pooley, G. G. 2003, MNRAS, 344, 60

Haardt, F., \& Maraschi, L. 1991, ApJ, 380, L51

Hawley, J. F., \& Balbus, S. A. 2002, ApJ, 573, 738

Heinz, S. 2004, MNRAS, in press (astro-ph/0409029)

Heinz, S., \& Sunyaev, R. 2003, MNRAS, 343, L59

Ho, L. C. 1999, ApJ, 516, 672

Ichimaru, S. 1977, ApJ, 214, 840

Igumenshchev, I. V., \& Abramowicz, M. A. 2000, ApJ, 537, L27

Igumenshchev, I. V., Narayan, R., \& Abramowicz, M. A. 2003, ApJ, 592, 2042

Kato, S., Fukue, J., \& Mineshige, S. 1998, Black Hole Accretion Disks (Kyoto Univ. Press)

Kato, S., Yamasaki, T. , Abramowicz, M. A., \& Chen, X. 1997, PASJ, 49, 221

Koratkar, A., \& Blaes, O. 1999, PASP, 111, 1

Lasota, J. P., Abramowicz, M. A., Chen, X., Krolik, J., Narayan, R., \& Yi, I. 1996, ApJ, 462, 142

Liu, B. F., Yuan, F., Meyer, F., Meyer-Hofmeister, E., \& Xie, G. Z. 1999, ApJ, 527, L17

Maccarone, T. 2003, A\&A, 409, 697

Maccarone, T. J., \& Coppi, P. S. 2003, MNRAS, 338, 189

Malkan, M. A. 1983, ApJ, 268, 582

Malzac, J., Merloni, A., \& Fabian, A. C. 2004, MNRAS, 351, 253

Maraschi, L., \& Tavecchio, F. 2003, ApJ, 593, 667 
Markoff, S., Falcke, H., \& Fender, R. P. 2001, A\&A, 372, L25

McClintock, J. E., Haswell, C. A., Garcia, M. R., Drake, J. J., Marshall, R. I., Muno, H. L., et al. 2001, ApJ, 555, 477

McClintock, J. E. \& Remillard, R. A. 2004, in Compact Stellar X-ray Sources, eds. W. H. G. Lewin, M. van der Klis (Cambridge Univ. Press), in press (astro-ph/0306213 v4)

Meier, D. L. 2001, ApJ, 548, L9

Merloni, A., Heinz, S., \& di Matteo, T. 2003, MNRAS, 345, 1057

Meyer, F., \& Meyer-Hofmeister, E. 1994, A\&A, 361, 175

Meyer-Hofmeister, E., Liu, B. F., \& Meyer, F. 2004, A\&A, in press (astro-ph/0411145)

Miyamoto, S., Kitamoto, S., Hayashida, K., \& Egoshi, W. 1995, ApJ, 442, L13

Nagar, N. M., Falcke, H., Wilson, A. S., \& Ho, L. C. 2000, ApJ, 542, 186

Nakamura, K. E., Kusunose, M., Matsumoto, R., \& Kato, S. 1997, PASJ, 49, 503

Narayan, R. 1996, ApJ, 461, 136

Narayan, R. 2002, in Lighthouses of the Universe, Eds. M. Gilfanov, R. Sunyaev (Springer)

Narayan, R., Barret, D., \& McClintock, J. E. 1997, ApJ, 482, 448

Narayan, R., Igumenshchev, \& Abramowicz, M. A. 2000, ApJ, 539, 798

Narayan, R., Mahadevan, R., \& Quataert, E. 1998, in The Theory of Black Hole Accretion Discs, eds. M. A. Abramowicz, G. Bjornsson, J. E. Pringle (Cambridge Univ. Press) p148

Narayan, R., \& McClintock, J. E. 2005, ApJ, submitted (astro-ph/0410556)

Narayan, R., McClintock, J. E., \& Yi, I. 1996, ApJ, 457, 821

Narayan, R., \& Yi, I. 1994, ApJ, 428, L13

Narayan, R., \& Yi, I. 1995a, ApJ, 444, 231

Narayan, R., \& Yi, I. 1995b, ApJ, 452, 710

Narayan, R., Yi, I., \& Mahadevan, R. 1995, Nature, 374, 623

Novikov, I. D., \& Thorne, K. S. 1973, in Blackholes, ed. C. DeWitt, B. DeWitt (Gordon \& Breach) p343

Nowak, M. A., Wilms, J., \& Dove, J. B. 2002, MNRAS, 332, 856

Pringle, J. E. 1976, MNRAS, 177, 65

Quataert, E. 2001, in Probing the Physics of Active Galactic Nuclei by Multiwavelength Monitoring, ed. B. M. Peterson, R. S. Polidan, R. W. Pogge (Astr. Soc. Pacific) p71

Quataert, E., di Matteo, T., Narayan, R., \& Ho, L. C. 1999, ApJ, 525, L89

Quataert, E., \& Gruzinov, A. 2000, ApJ, 539, 809

Quataert, E., \& Narayan, R. 1999, ApJ, 520, 298

Rees, M. J., Begelman, M. C., Blandford, R. D., \& Phinney, E. S. 1982, Nature, 295, 17

Reynolds, C. S., di Matteo, T., Fabian, A. C., Hwang, U., \& Canizares, C. R. 1996, MNRAS, 283, L111

Rozanska, A., \& Czerny, B. 2000, A\&A, 360, 1170

Shakura, N. I., \& Sunyaev, R. A. 1973, A\&A, 24, 337

Shapiro, S. L., Lightman, A. P., \& Eardley, D. M. 1976, ApJ, 204, 187

Spruit, H. C., \& Deufel, B. 2002, A\&A, 387, 918

Stone, J. M., \& Pringle, J. E. 2001, MNRAS, 322, 461

Stone, J. M., Pringle, J. E., \& Begelman, M. C. 1999, MNRAS, 310, 1002

Tananbaum, H., Gursky, H., Kellogg, E., Giacconi, R., \& Jones, C. 1972, ApJ, 177, L5

Wu, X. B. 1997, MNRAS, 292, 113 
Yuan, F. 2001, MNRAS, 324, 119

Yuan, F., Cui, W., \& Narayan, R. 2004, ApJ, in press (astro-ph/0407612)

Yuan, F., \& Narayan, R. 2004, ApJ, 612, 724

Yuan, F., Quataert, E., \& Narayan, R. 2003, ApJ, 598, 301

Yuan, F., \& Zdziarski, A. A. 2004, MNRAS, 354, 953

Zdziarski, A. A., Gierlinski, M., Mikolajewska, J., Wardzinski, G., Smith, D. M., Alan, H. B., \& Kitamoto, S. 2004, MNRAS, 351, 791

Zdziarski, A. A., Lubinski, P., Gilfanov, M., \& Revnivtsev, M. 2003, MNRAS, 342, 355

Address for Offprints: Kluwer Prepress Department

P.O. Box 990

3300 AZ Dordrecht

The Netherlands 